\title{
ON THE NEWTON POLYTOPE
}

\section{ALVIN I. THALER}

1. Introduction. The theory of the Newton polygon of a polynomial in one variable with coefficients in a complete non-Archimedean valued field is well known (see, for example, [1], [2], [3], [6]). In [4], Krasner states that one may construct an analogous Newton polytope for a polynomial in several variables. In this paper we explore the properties of the Newton polytope.

I am very grateful to Professor B. M. Dwork for his encouragement and advice.

2. Preliminaries. Let $K$ be a complete field with respect to a nonArchimedean rank one valuation $x \rightarrow \operatorname{ord} x$ with value group $B \subset R$, where $R$ denotes the additive group of real numbers. We shall assume that $B$ is dense in $R$. Let $\Re$ be the algebraic closure of $K$, and extend the valuation to $\Omega$ in the natural manner. As in [2], for each real number $b$ we define $\Gamma_{b}=\{\xi \in \Omega$ : ord $\xi=b\}$.

Definition 1. Let $f(x)=\sum_{i=0}^{n} a_{i} x^{i} \in K[x]$. For any $\mu \in R, v(f ; \mu)$ $=\operatorname{Min}_{0 \leq i \leq n}\left(\right.$ ord $\left.a_{i}+i \mu\right)$.

REMARK. $v(f ; \mu)$ is the $Y$-intercept of the lower line of support of the Newton polygon of $f$ with slope $-\mu$.

We need the following results from the one-variable theory.

Proposition 1. Let $f(x) \in K[x]$ have a zero on $\Gamma_{r}$.Then for any $\left.\lambda \in B\right)$ satisfying the inequality $\lambda \geqq v(f ; r)$, there exists $\xi \in \Gamma_{r}$ such that ord $f(\xi)$ $=\lambda$.

Proof. (a) If $a_{0} \neq 0$ and $-r$ is the slope of the first side of the Newton polygon of $f$ (i.e., if, for all $r^{\prime}>r, f$ has no zero on $\Gamma_{r^{\prime}}$ ) then clearly $v(f ; r)=\operatorname{ord} a_{0}$. Therefore, we need only choose $\gamma \in \Gamma_{\lambda}$ such that ord $\left(a_{0}-\gamma\right)=$ ord $a_{0}$, for then the polynomials $f(x)$ and $f(x)-\gamma$ will have identical Newton polygons. If $\lambda>v(f ; r)$, then for any $\gamma \in \Gamma_{\lambda}$, ord $\left(a_{0}-\gamma\right)=\operatorname{ord} a_{0} ;$ if $\lambda=v(f ; r)$, we choose $\alpha, \beta \in \Gamma_{0}$ such that $\alpha+\beta \in \Gamma_{0}$ (this can be done since the residue class field of $\Omega$ contains more than two elements), and put $\gamma=a_{0}\left(1+\alpha \beta^{-1}\right)$.

(b) If either $a_{0}=0$ or $-r$ is not the slope of the first side of the Newton polygon of $f$, let $\gamma$ be any element of $\Gamma_{\lambda}$, and consider the Newton diagram of $f(x)-\gamma$ : clearly the Newton diagram of $f(x)-\gamma$ coincides with the Newton diagram of $f(x)$, with the possible excep-

Received by the editors July 29, 1963. 
tion of the points with zero abscissa. Since ord $\left(a_{0}-\gamma\right) \geqq v(f ; r),-r$ is the slope of a side of the Newton polygon of $f(x)-\gamma$.

The following result is essentially identical to Lemma 1.2 of [2].

LEMma 1. Let $f_{1}(x), f_{2}(x), \cdots, f_{n}(x)$ be a finite set of polynomials with coefficients in $K$, let $\rho \in(3)$. Then there exists $\xi \in \Gamma_{\rho}$ such that ord $f_{i}(\xi)=v\left(f_{i} ; \rho\right), i=1,2, \cdots, n$.

Proof. Let $v\left(f_{i} ; \rho\right)=M_{i}, i=1,2, \cdots, n$; then $M_{i} \in$ (5). Therefore, we may choose $\pi_{i} \in \Gamma_{M_{i}}, \pi \in \Gamma_{\rho}$. For each $i$, we put $g_{i}(x)=f_{i}(\pi x) / \pi_{i}$. Then the coefficients of $g_{i}(x)$ are integral and the image of $g_{i}(x)$ in the residue class field of $\Omega$ is nontrivial. Since the residue class field is infinite there is a unit $\xi^{\prime}$ in $\Re$ such that ord $g_{i}\left(\xi^{\prime}\right)=0, i=1,2, \cdots$, $n$. If we put $\xi=\pi \xi^{\prime}$, we have the desired result.

3. The Newton polytope. Let $f(x, y)=\sum a_{i j} x^{i} y^{j} \in K[x, y]$. The point set $\left\{\left(i, j\right.\right.$, ord $\left.\left.a_{i j}\right)\right\}$ is called the Newton diagram of $f(x, y)$. We define the convex closure of the Newton diagram of $f(x, y)$ with the point $(0,0,+\infty)$ to be the Nerwton polytope of $f(x, y)$. tion

REMARK. The Newton polytope of $f(x, y)$ is the graph of the func-

$$
\mathbf{\Pi}_{f}(X, Y)=\operatorname{Sup}_{\mu, \nu \in R}[\nu(f ; \mu, \nu)-\mu X-\nu Y],
$$

where $v(f ; \mu, \nu)$ is defined in the obvious manner generalizing Definition 1: $v(f ; \mu, \nu)=\operatorname{Min}_{i, j}\left(\right.$ ord $\left.a_{i j}+i \mu+j \nu\right)$ (see [5, p. 49]).

Let $(\xi, \eta) \in \Omega \times \Omega$, suppose $(\xi, \eta) \in \Gamma_{\rho} \times \Gamma_{\sigma}$. The following result gives an estimate for ord $f(\xi, \eta)$ in terms of $\rho, \sigma$.

Proposition 2. Let $P$ be the lower plane of support of the Newton polytope of $f(x, y)$, with $\partial Z / \partial X=-\rho, \partial Z / \partial Y=-\sigma$. Suppose $(\xi, \eta)$ $\in \Gamma_{p} \times \Gamma_{\sigma}$. If only one vertex of the polytope lies on $P$, then only one term of $f(\xi, \eta)$ attains minimal ord, and then ord $f(\xi, \eta)=v(f ; \rho, \sigma)$, the $Z$-axis intercept of $P$. Otherwise, ord $f(\xi, \eta) \geqq v(f ; \rho, \sigma)$.

Proof. Let the plane $P_{i j}$ be defined by the equation $Z+\rho X+\sigma Y$ $=\operatorname{ord}\left(a_{i j} \xi^{\xi} \eta^{j}\right)$. Then the point $\left(i, j\right.$, ord $\left.a_{i j}\right)$ in the Newton diagram of $f(x, y)$ lies in $P_{i j}$; but $\operatorname{ord}\left(a_{i j} \xi^{i} \eta^{i}\right)<\operatorname{ord}\left(a_{i^{\prime} j^{\prime}} \xi^{i^{\prime}} \eta^{j^{\prime}}\right)$ (respectively $\left.\operatorname{ord}\left(a_{i j} \xi^{i} \eta^{j}\right) \leqq \operatorname{ord}\left(a_{i^{\prime} j^{\prime}} \xi^{\xi^{\prime}} \eta^{j^{\prime}}\right)\right)$ if and only if the intercept cut off on the $Z$-axis by the plane $P_{i j}$ is less than (respectively less than or equal to) that cut off by $P_{i^{\prime} j^{\prime}}$. Thus, ord $\left(a_{i_{0} j_{0}} \xi^{i_{0}} \eta^{j 0}\right)=\operatorname{Min}_{i, j} \operatorname{ord}\left(a_{i j} \xi^{i} \eta^{i}\right)$ if and only if $P_{i_{0} j_{0}}$ is the lower plane of support of the Newton polytope with $\partial Z / \partial X=-\operatorname{ord} \xi, \partial Z / \partial Y=-\operatorname{ord} \eta$.

Corollary. If $(\xi, \eta)$ is a zero of $f(x, y)$, then the lower plane of sup- 
port $P$ of the Newton polytope of $f(x, y)$ with $\partial Z / \partial X=-\operatorname{ord} \xi, \partial Z / \partial Y$ $=-$ ord $\eta$ contains an edge of the polytope.

REMARK. No distinction is made here between the plane $P$ containing an edge or a face of the polytope.

The converse to the corollary of Proposition 2 is also valid. Thus, the Newton polytope of $f(x, y)$ gives an explicit criterion for determining the existence of a zero of $f(x, y)$ on $\Gamma_{r} \times \Gamma_{s}$. Before proceeding to the proof of the converse, we introduce the following notation.

Let $f(x, y)=f_{0}(x)+f_{1}(x) y+f_{2}(x) y^{2}+\cdots+f_{n}(x) y^{n}, f_{i}(x) \in K[x]$, $i=0,1,2, \cdots, n$. We shall assume that $f(x, y) \notin K[x], f_{n}(x) \neq 0$. Let II denote the Newton polytope of $f(x, y)$. For $\rho \in(\$)$, let $\Lambda_{\rho}$ be the convex closure in the $Y Z$-plane of the point set $\left\{\left(0, j, v\left(f_{j} ; \rho\right)\right)\right.$ : $j=0,1,2, \cdots, n\}$ with the point $(0,0,+\infty)$. For $\xi \in K$, let $\Lambda_{\xi}$ be the convex closure in the $Y Z$-plane of the point set $\left\{\left(0, j\right.\right.$, ord $\left.f_{j}(\xi)\right)$ : $j=0,1,2, \cdots, n\}$ with the point $(0,0,+\infty)$. We observe that $\Lambda_{\xi}$ is the Newton polygon of the polynomial $g_{\xi}(y)=\sum f_{j}(\xi) y^{i}$, and that if ord $\xi=\rho$, then no point of $\Lambda_{\rho}$ lies below $\Lambda_{\xi}$. Let $\Pi_{j}$ denote the Newton polygon of the polynomial $f_{j}(x)$ in the plane $Y=j$, and finally let $l_{j}(\rho)$ be the lower line of support of $\Pi_{j}$ with slope $-\rho$ in the plane $Y=j$.

Proposition 3. Let $f(x, y) \in K[x, y]$, let $r, s \in\left(S\right.$. Suppose $P_{r s}$ is the lower plane of support of $\mathbf{\Pi}$, the Newton polytope of $f(x, y)$, with equation $Z+r X+s Y+d=0$. If $P_{r s}$ contains an edge of $\mathbf{\Pi}$, then there is a point $(\xi, \eta) \in \Gamma_{r} \times \Gamma_{s}$ such that $f(\xi, \eta)=0$.

Proof. Suppose $P_{r s}$ contains an edge of $\boldsymbol{\Pi}$ with direction numbers $(\alpha, \beta, \gamma)$. Since $P_{r s}$ cannot contain a vertical line, either $\alpha$ or $\beta$ is different from zero. We may assume, with no loss of generality, that $\beta \neq 0$. Then a pair of points $p_{1}=\left(i_{1}, j_{1}\right.$, ord $\left.a_{i_{1} j_{1}}\right), p_{2}=\left(i_{2}, j_{2}\right.$, ord $\left.a_{i_{2} j_{2}}\right)$ of the Newton diagram of $f(x, y)$ is on $P_{r s}$, with $j_{1} \neq j_{2}$. Since $P_{r s}$ is a lower plane of support of $\boldsymbol{\Pi}$ containing $p_{1}$ and $p_{2}$, with $\partial Z / \partial X=-r$, it follows that $l_{j_{1}}(r)$ and $l_{j_{2}}(r)$ are in $P_{r s}$. By Lemma 1 , we may choose $\xi \in \Gamma_{r}$ such that ord $f_{j_{1}}(\xi)=v\left(f_{j_{1}} ; r\right)$, ord $f_{j_{2}}(\xi)=v\left(f_{j_{2}} ; r\right)$. Thus, the points $q_{1}=\left(0, j_{1}\right.$, ord $\left.f_{j_{1}}(\xi)\right)$ and $q_{2}=\left(0, j_{2}\right.$, ord $\left.f_{j_{2}}(\xi)\right)$ of the Newton diagram of $g_{\xi}(y)$ are in $P_{r_{s}}$, and are therefore on a side of $\Lambda_{\xi}$ which lies in $P_{r s}$ (since no point of $\Lambda_{\xi}$ can lie below the intersection of $P_{r s}$ with the $Y Z$-plane). But since $\Lambda_{\xi}$ lies in the $(X=0)$-plane, we see that the side of $\Lambda_{\xi}$ determined by $q_{1}, q_{2}$ has slope $\partial Z / \partial Y=-s$; therefore, the polynomial $g_{\xi}(y)$ has a root $\eta \in \Gamma_{s}$. Hence, $(\xi, \eta) \in \Gamma_{r} \times \Gamma_{s}$ and $f(\xi, \eta)=0$.

We summarize these results in 
Theorem 1. Let $f(x, y) \in K[x, y]$, let $r, s \in \mathbb{G}$, and let $P_{r s}$ be the lower plane of support of the Newton polytope of $f(x, y)$ with $\partial Z / \partial X=-r$, $\partial Z / \partial Y=-s$. There is a zero $(\xi, \eta)$ of $f(x, y)$ such that ord $\xi=-r$, ord $\eta=-s$ if, and only if, the plane $P_{r s}$ contains an edge of the polytope.

\section{Distinguished values.}

Definition 2. Let $D$ be a subset of $\Omega \times \Omega$, let $r$ (respectively $s$ ) be a real number. We say that $r$ is $x$-distinguished on $D$ (respectively, $s$ is $y$-distinguished on $D$ ) if there are infinitely many $s \in \mathbb{S}$ (respectively, infinitely many $r \in(S)$ such that $D \cap\left(\Gamma_{r} \times \Gamma_{s}\right) \neq \varnothing$.

Proposition 4. Let $f(x, y) \in K[x, y]$, suppose $f(x, y) \neq 0 ;$ let $D=V(f)$ $=\{(\xi, \eta) \in \Omega \times \Omega: f(\xi, \eta)=0\}$. The set of real numbers which are $x$ distinguished on $D$ (respectively, $y$-distinguished on $D$ ) is finite.

Proof. Let $f(x, y)=f_{0}(x)+f_{1}(x) y+f_{2}(x) y^{2}+\cdots+f_{n}(x) y^{n}, f_{i}(x)$ $\in K[x], 0 \leqq i \leqq n$. Since $f(x, y) \neq 0$, not all the polynomials $\left\{f_{i}(x)\right\}$ are zero. Let $\mathfrak{F}$ be the subset of $\left\{f_{i}(x): 0 \leqq i \leqq n\right\}$ consisting of those polynomials which are nonzero, and let $\Re$ be the set of values of zeros of polynomials in $\mathfrak{F}$, i.e., $r \in \Re$ if there is a pair $(f, \xi) \in \mathfrak{F} \times \Gamma_{r}$ such that $f(\xi)=0$. Clearly $\Re$ is a finite set. Suppose $r^{\prime} \notin \Re$. Then for $\xi \in \Gamma_{r^{\prime}}$, the Newton diagram of $g_{\xi}(y)=f(\xi, y)$ depends only on ord $\xi$. Therefore, as $\xi$ runs through $\Gamma_{r^{\prime}}$, there is only a finite number of $s \in \&$ such that $g_{\xi}$ has a zero on $\Gamma_{s}$. Therefore if $r^{\prime} \notin \Re, r^{\prime}$ is not $x$-distinguished on $D$.

The set of real numbers which are distinguished for a given polynomial is determined by the Newton polytope of that polynomial. In fact, we shall prove

TheOREM 2. Let $f(x, y) \in K[x, y]$, suppose $f(0,0) \neq 0$. Then $\rho$ is $x$-distinguished on $V(f)$ if, and only if, there is an edge of the Newton polytope of $f(x, y)$ with direction numbers $(1,0,-\rho)$.

The proof of Theorem 2 will be a trivial consequence of Propositions 5 and 6.

Lemma 2. Let $f(x, y)=f_{0}(x)+f_{1}(x) y+f_{2}(x) y^{2}+\cdots+f_{n}(x) y^{n}$ $\in K[x, y]$, suppose $f(x, y) \notin K[x], f(0,0) \neq 0$. Let $\rho \in \$\left(\right.$ and let $\Lambda_{\rho}, \Pi_{j}$, $0 \leqq j \leqq n$, be as previously defined. If the point $\left(0, j_{0}, v\left(f_{j_{0}} ; \rho\right)\right)$ is on $\Lambda_{\rho}$, then there is a vertex $\left(i_{0}, j_{0}\right.$, ord $\left.a_{i_{0} j_{0}}\right)$ of $\Pi_{j_{0}}$ which is on the Newton polytope of $f(x, y)$.

Proof. Suppose the point $\left(0, j_{0}, v\left(f_{j_{0}} ; \rho\right)\right)$ is on the side of $\Lambda_{\rho}$ with vertices $\left(0, j_{1}, v\left(f_{j_{1}} ; \rho\right)\right),\left(0, j_{2}, v\left(f_{j_{2}} ; \rho\right)\right)$, and suppose $j_{1}<j_{2}$. Let $P$ be the plane determined by the (parallel) lines $l_{j_{1}}(\rho)$ and $l_{j_{2}}(\rho)$. Then certainly $l_{j_{0}}(\rho)$ lies in $P$. It remains only to be shown that $P$ is a 
lower plane of support of the polytope. Suppose not; then there is a point $\left(i^{\prime}, j^{\prime}\right.$, ord $\left.a_{i^{\prime} j^{\prime}}\right)$ below $P$. Hence $\left(0, j^{\prime}, v\left(f_{j^{\prime}} ; \rho\right)\right)$ lies below the line $P \cap(X=0)$. This contradicts convexity of $\Lambda_{\rho}$ in the $Y Z$-plane.

Corollary. Using the above notation, if $\left(0, j_{0}, v\left(f_{j_{0}} ; \rho\right)\right)$ is on $\Lambda_{p}$, and if ord $f_{j_{0}}(\xi)$ has more than one value for $\xi \in \Gamma_{\rho}$, then a side of $\Pi_{j_{0}}$ is on the polytope of $f(x, y)$.

Proposition 5. If $\rho \in(S)$ is $x$-distinguished on $V(f)$, then there is an edge of the Newton polytope of $f$ with direction numbers $(1,0,-\rho)$.

Proof. For $\xi \in \Gamma_{\rho}$, we let $g_{\xi}(y), \Lambda_{\xi}, \Lambda_{\rho}$ be defined as before. Since $\rho$ is $x$-distinguished on $V(f)$, the set of slopes of sides of the polygons $\left\{\Lambda_{\xi}: \xi \in \Gamma_{\rho}\right\}$ is infinite. Consider the set of non-negative integers $j$ with the property that $\left(0, j, v\left(f_{j} ; \rho\right)\right)$ is a vertex of $\Lambda_{\rho}$ and \{ord $f_{j}(\xi): \xi \in \Gamma_{\rho}$ \} has more than one element. If this set were empty, it would follow that $\Lambda_{\rho}=\Lambda_{\xi}$ for each $\xi \in \Gamma_{\rho}$, contradicting the hypothesis. Let $j_{0}$ denote the smallest integer of this set.

By the previous corollary, $l_{j_{0}}(\rho)$ contains a side of $\Pi_{j_{0}}$, and this side is on $\mathbf{\Pi}$. To complete the proof of Proposition 5, we need only show that this side of $\Pi_{j_{0}}$ is indeed an edge of the polytope. If $j_{0}$ is either 0 or $n$, this is certainly the case. Otherwise, we may choose integers $j_{1}, j_{2}$ such that $\left(0, j_{1}, v\left(f_{j_{1}} ; \rho\right)\right),\left(0, j_{0}, v\left(f_{j_{0}} ; \rho\right)\right)$ and $\left(0, j_{2}, v\left(f_{j_{2}} ; \rho\right)\right)$ are distinct adjacent vertices of $\Lambda_{\rho}$, with $0 \leqq j_{1}<j_{0}<j_{2} \leqq n$. Let $P_{1}$ be the plane determined by the lines $l_{j_{0}}(\rho), l_{j_{1}}(\rho)$, and let $P_{2}$ be the plane determined by the lines $l_{j_{0}}(\rho), l_{j_{2}}(\rho)$. By the concluding argument of Lemma 2, $P_{1}$ and $P_{2}$ are lower planes of support of the Newton polytope of $f(x, y)$. By choice of $j_{1}$ and $j_{2}$, they are distinct, and their intersection is the line $l_{j_{0}}(\rho)$. This completes the proof.

Proposition 6. If there is an edge of the Newton polytope of $f(x, y)$ with direction numbers $(1,0,-\rho)$, then $\rho \in ß$ and $\rho$ is $x$-distinguished on $V(f)$.

Note. It is not necessary to assume here that $\rho \in(5)$.

Proof. We again write $f(x, y)=\sum_{j=1}^{n} f_{j}(x) y^{j}$; what we are required to show is that, if there is a polynomial $f_{i}(x)$ such that $f_{i}(x)$ has a zero on $\Gamma_{\rho}$ and, moreover, that the side of $\Pi_{i}$ of slope $-\rho$ is an edge of the Newton polytope $\boldsymbol{\Pi}$ of $f(x, y)$, then $\rho$ is $x$-distinguished on $V(f)$. That is, we must show that the set $\ell_{\rho}=\left\{\lambda:-\lambda\right.$ is the slope of a side of $\Lambda_{\xi}$, for some $\left.\xi \in \Gamma_{\rho}\right\}$ is infinite. (We observe that $\rho \in \mathcal{H}$, from the onevariable Newton polygon theory applied to $f_{i}(x)$.)

Case 1. For some $k, 0 \leqq k \leqq n, f_{k}$ has no zeros on $\Gamma_{\rho}$. Let $k_{0}$ be the smallest such $k$. Then either $k_{0}=0$ or $k_{0}>0$. 
(1a) Suppose $k_{0}=0$. Let $i_{0}$ be the smallest integer with the property that a side of $\Pi_{i_{0}}$ of slope $-\rho$ is an edge of $\Pi$. Then $\left(0, i_{0}, v\left(f_{i_{0}} ; \rho\right)\right)$ is a vertex of $\Lambda_{\rho}$. Moreover, $i_{0}>0$, since $f_{0}$ has no zeros on $\Gamma_{\rho}$.

Let the vertices of $\Lambda_{\rho}$ in the $Y Z$-plane have $Y$-coordinates $0=\alpha_{0}$ $<\alpha_{1}<\cdots<\alpha_{t}$, let $i_{0}=\alpha_{\imath}$. Then for all $\xi \in \Gamma_{\rho}$, the polygons $\Lambda_{\xi}$ and $\Lambda_{\rho}$ agree in vertices whose $Y$-coordinates are $\alpha_{0}, \alpha_{1}, \cdots, \alpha_{\iota-1}$.
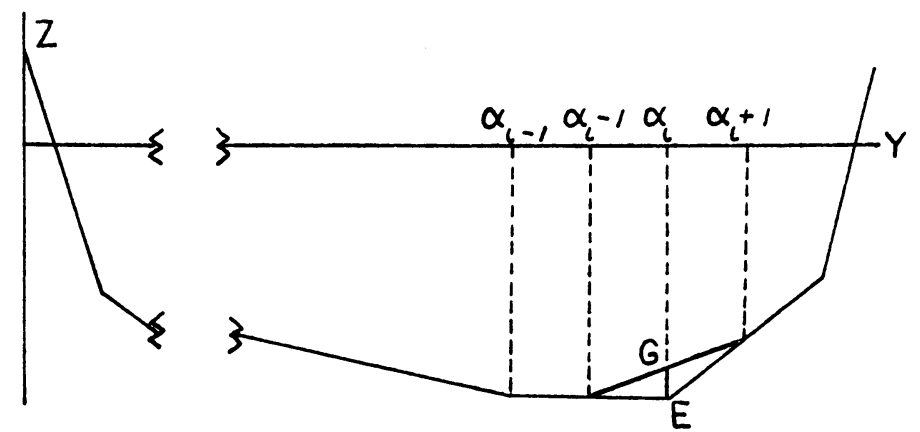

FIGURE 1. Newton polygon of $\Lambda_{\boldsymbol{\rho}}$.

Consider the set $B$ of $Z$-coordinates of points on the line $\overline{E G}$ in Figure 1 which are also in $\left(S .{ }^{1}\right.$ Since $(B)$ is dense in $R$, the set $B$ is infinite. But $E$ has coordinates $\left(i_{0}, v\left(f_{i_{0}} ; \rho\right)\right)$, whence from Proposition 1 , we may choose, for each $r \in \mathcal{Z}$, an element $\xi_{r} \in \Gamma_{\rho}$ such that ord $f_{i_{0}}\left(\xi_{r}\right)$ $=r$. Let $\Xi$ be a set of representatives of $及$ in $\Gamma_{\rho}$ : if $\xi \in \Xi$ then ord $f_{i_{0}}(\xi)$ $\in \Omega$, and $\xi, \xi^{\prime} \in \Xi, \xi \neq \xi^{\prime}$ implies ord $f_{i_{0}}(\xi) \neq$ ord $f_{i_{0}}\left(\xi^{\prime}\right)$. The definition of 3 guarantees that $\left(\alpha_{\iota}\right.$, ord $\left.f_{\alpha_{\iota}}(\xi)\right)$ is a vertex of $\Lambda_{\xi}$ for any $\xi \in \Xi$.

For $\alpha_{\imath-1} \leqq \nu \leqq \alpha_{\imath}-1$, we define the sets $\Xi_{\nu}^{(1)}=\left\{\xi \in \Xi:\left(\nu\right.\right.$, ord $\left.f_{\nu}(\xi)\right)$, $\left(i_{0}\right.$, ord $\left.f_{i_{0}}(\xi)\right)$ are vertices of a side of $\left.\Lambda_{\xi}\right\}$. Then, since $\Xi=U_{\alpha_{l-1} \leq \alpha_{\imath}-1} \Xi_{\nu}^{(1)}$, we may choose $\nu_{1}$ to be the largest integer with the property that $\nu_{1}<i_{0}$ and $\Xi_{\nu_{1}}^{(1)}$ is an infinite set. Let $\mathfrak{T}_{1}=\left\{\right.$ ord $f_{\nu_{1}}(\xi)$ : $\left.\xi \in \Xi_{\nu_{1}}^{(1)}\right\}$. If $\mathfrak{T}_{1}$ is finite, then the set $\left\{\right.$ ord $\left.f_{\nu_{1}}(\xi)-\operatorname{ord} f_{i_{0}}(\xi): \xi \in \Xi_{\nu_{1}}^{(1)}\right\}$ is infinite, whence so is $\mathfrak{R}_{p}$. Otherwise we define, for $\alpha_{\imath-1} \leqq \nu<\nu_{1}$, the sets $\Xi_{\nu}^{(2)}=\left\{\xi \in \Xi_{\nu_{1}}^{(1)}:\left(\nu\right.\right.$, ord $\left.f_{\nu}(\xi)\right),\left(\nu_{1}\right.$, ord $\left.f_{\nu_{1}}(\xi)\right)$ are vertices of a side of $\left.\Lambda_{\xi}\right\}$, and choose $\nu_{2}$ to be the largest integer with the property that $\nu_{2}<\nu_{1}$ and $\Xi_{\nu_{2}}^{(2)}$ is an infinite set. We then define $\mathfrak{I}_{2}=\left\{\right.$ ord $\left.f_{\nu_{2}}(\xi): \xi \in \Xi_{\nu_{2}}^{(2)}\right\}$. Proceeding in this manner, we define a sequence of integers $\nu_{1}>\nu_{2}$ $>\cdots>\nu_{m} \geqq \alpha_{\iota-1}$, and a corresponding sequence of sets $\mathfrak{I}_{1}, \mathfrak{T}_{2}, \cdots$, $\mathfrak{T}_{m}$. If $\mathfrak{T}_{i}$ is finite for some $i$, then (1a) is proved. Otherwise, we may assume that $m$ is such that $\nu_{m}=\alpha_{\iota-1}$; but then, $\mathfrak{I}_{m}=\left\{\operatorname{ord} f_{\alpha_{\iota-1}}(\xi)\right.$ : $\left.\xi \in \Xi_{\alpha_{\iota-1}}^{(m)}\right\}=\left\{v\left(f_{\alpha_{\imath-1}} ; \rho\right)\right\}$, which is certainly finite. Thus, case (1a) is proved.

1 If $\alpha_{\imath}=n$, let $B=\left\{\lambda \in(B)\right.$ ord $\left.\lambda \geqq v\left(f_{n} ; \rho\right)\right\}$. 
(1b) Suppose $k_{0}>0$. Then $f_{0}$ has a zero on $\Gamma_{\rho}$. Hence, by Proposition 1 , we may choose a sequence $\left\{\xi_{h}\right\} \subset \Gamma_{\rho}$ such that ord $f_{0}\left(\xi_{h}\right) \rightarrow \infty$ as $h \rightarrow \infty$. If $\rho$ is not $x$-distinguished on $V(f)$, we must then have ord $f_{1}\left(\xi_{h}\right) \rightarrow \infty$ as $h \rightarrow \infty, \cdots$, ord $f_{k_{0}-1}\left(\xi_{h}\right) \rightarrow \infty$ as $h \rightarrow \infty$. But then, for $h$ sufficiently large, the point $\left(k_{0}\right.$, ord $\left.f_{k_{0}}\left(\xi_{h}\right)\right)=\left(k_{0}, v\left(f_{k_{0}} ; \rho\right)\right)$ is on $\Lambda_{\xi_{h}}$.

For $0 \leqq \nu<k_{0}$, we let $\mathcal{E}_{\nu}=\left\{h \in Z:\left(\nu\right.\right.$, ord $\left.f_{\nu}\left(\xi_{h}\right)\right),\left(k_{0}\right.$, ord $\left.f_{k_{0}}\left(\xi_{h}\right)\right)$ are vertices of a side of $\left.\Lambda_{\xi_{h}}\right\}$. Then for some $\nu_{0}$, the set $\varepsilon_{\nu_{0}}$ is infinite, whence the set of slopes

$$
\left\{\frac{v\left(f_{k_{0}} ; \rho\right)-\operatorname{ord} f_{\nu_{0}}\left(\xi_{h}\right)}{k_{0}-\nu_{0}}: h \in \varepsilon_{v_{0}}\right\}
$$

is infinite, whence $\rho$ is $x$-distinguished on $V(f)$.

Case 2. $f_{j}(x)$ has a zero on $\Gamma_{\rho}$ for each $j, 0 \leqq j \leqq n$. If there is an $\eta \in \Gamma_{\rho}$ such that $f_{j}(\eta)=0$ for each $j, 0 \leqq j \leqq n$, then certainly $\rho$ is $x$ distinguished on $V(f)$. Therefore, we may assume that no root of one of the $f_{j}$ is a root of all the $f_{j}$.

Let $f_{0}$ have the zeros $\eta_{1}, \eta_{2}, \cdots, \eta_{w}$ on $\Gamma_{\rho}$. Let $j_{0}$ be the smallest integer with the property that, for some $i_{0}, 1 \leqq i_{0} \leqq w, f_{j_{0}}\left(\eta_{i_{0}}\right) \neq 0$. Choose a neighborhood $N$ of $\eta_{i_{0}}$ in $\Omega$ such that $\left\{\right.$ ord $\left.f_{j_{0}}(\xi): \xi \in N\right\}$ is bounded. Since the valuation of $\Omega$ is dense, we may choose a sequence $\left\{\xi_{h}\right\} \subset N$ such that ord $f_{k}\left(\xi_{h}\right) \rightarrow \infty, h \rightarrow \infty$, for $k=0,1,2, \cdots, j_{0}-1$. But ord $\left.f_{j_{0}}\left(\xi_{h}\right): h=1,2, \cdots\right\}$ is bounded; therefore, for $h$ sufficiently large, the point $\left(j_{0}\right.$, ord $\left.f_{j_{0}}\left(\xi_{h}\right)\right)$ is on $\Lambda_{\xi_{h}}$.

For $0 \leqq \nu<j_{0}$, let $\varepsilon_{\nu}=\left\{h \in Z:\left(\nu\right.\right.$, ord $\left.f_{\nu}\left(\xi_{h}\right)\right),\left(j_{0}\right.$, ord $\left.f_{\nu_{0}}\left(\xi_{h}\right)\right)$ are vertices of a side of $\left.\Lambda_{\xi_{h}}\right\}$. Then for some $\nu_{0}, \varepsilon_{\nu_{0}}$ is infinite; therefore, the set of slopes

$$
\left\{\frac{\operatorname{ord} f_{j_{0}}\left(\xi_{h}\right)-\operatorname{ord} f_{\nu_{0}}\left(\xi_{h}\right)}{j_{0}-\nu_{0}}: h \in \mathcal{E}_{\nu_{0}}\right\}
$$

is infinite, whence $\rho$ is $x$-distinguished on $V(f)$.

\section{REFERENCES}

1. E. Artin, Algebraic numbers and algebraic functions. I, Mimeographed notes, Princeton University, Princeton, N. J., 1951.

2. B. Dwork, On the zeta function of a hypersurface, Inst. Hautes Etudes Sci. Publ. Math. No. 12 (1962), 5-68.

3. M. Krasner, Theorie des fonctions, C. R. Acad. Sci. Paris 222 (1946), 37-40.

4. - Théorie des fonctions, C. R. Acad. Sci. Paris 222 (1946), 582.

5. M. Lazard, Les zeros des fonctions analytiques d'une variable sur un corps value complet, Inst. Hautes Etudes Sci. Publ. Math. No. 14 (1962), 47-75.

6. A. Ostrowski, Untersuchungen zur arithmetischen Theorie der Körper, Math. Z. 39 (1934-35), 269-320.

JoHNS Hopkins UNIVERSITY 